\title{
Effect of Surface Treatments on Viscoelastic Measurements of Thread-like Micellar Solutions
}

\author{
Tadashi InOuE $^{* \dagger}$, Ryo NAKATSUJI ${ }^{* *}$, Hiroshi WATANABE ${ }^{* *}$, and Yoshinobu TsuJII ${ }^{* *}$ \\ *Graduate School of Science, Osaka University, Toyonaka, Osaka 560-0043, JAPAN \\ ${ }^{* *}$ Institute for Chemical Research, Kyoto University, Uji, Kyoto 611-0011, JAPAN
}

(Received : April 1, 2008)

\begin{abstract}
Thread-like micelles are formed in aqueous solutions of cationic surfactants and a certain type of salts, and for some of these solutions, the strain hardening behavior is observed after inception of shear flow at high shear rates. In the present study, we examined the effect of chemical surface treatments of the cone-and-plate fixture made of glass on measurements of strain hardening behavior of the thread-like micellar solutions. The untreated glass fixture gave significantly weak strain hardening behavior, when compared with the ordinary stainless steel fixture. This could be attributed to the slippage of the solution on the surface of fixture. When the surface of glass fixture was modified with trimethychlorosilane to increase the dispersion component of surface free energy, the pronounced strain hardening was observed. Thus, the surface treatment of rheometer fixtures is very important to examine the nonlinear rheology of thread-like micellar solutions.
\end{abstract}

Key Words: Thread-like micelles / Strain hardening / Surface treatment / Flow instability

\section{INTRODUCTION}

Aqueous solutions of cationic surfactants form threadlike micelles and show pronounced viscoelastic behavior at low concentrations when certain aromatic acids or salts are added. One of the well-known examples is aqueous solutions of cetyltrimethylammonium bromide (CTAB) and sodium salicylate (NaSal). ${ }^{1-3)}$ Thread-like micelles can densely entangle with each other and show various types of nonlinear rheology. Examples of complicated nonlinear rheology are the shear-induced structure formation such as shear banding and shear-induced phase separation. In order to analyze the shear-induced structures, various optical methods such as flow birefringence ${ }^{4-6)}$, flow light scattering ${ }^{7-9)}$, and flow visualization $^{10,11)}$ have been widely utilized. These apparatus normally have the glass windows to transmit the light. Recently, we realized that measurement of nonlinear rheology of thread-like micelles is strongly affected by the material of the rheometer fixtures. In addition, for the case of glass fixture, we found that the surface treatment of the glass has a significant effect on the nonlinear viscoelastic data. In this paper, we report the effect of surface treatments of the glass cone-plate fixture on strain-hardening behavior of CTAB/NaSal solution.

† To whom all correspondence should be addressed

tadashi@chem.sci.osaka-u.ac.jp

\section{EXPERIMENTAL}

Cetyltrimethylammonium bromide (CTAB) and sodium salicylate (NaSal), were purchased from Wako Pure Chemical Industries Ltd. CTAB was purified by following a reported method. ${ }^{1,12)}$ Concentration of CTAB, $C_{\mathrm{D}}$, and concentration of NaSal, $C_{\mathrm{S}}$, were fixed at $C_{\mathrm{D}}=0.1 \mathrm{M}$ and $C_{\mathrm{S}}=0.1 \mathrm{M}$. All solutions were kept quiescently at room temperature prior to the tests for at least for two days for equilibration.

All viscoelastic data were obtained by using Rheometric ARES system with the cone-plate geometry. Glass fixtures finely polished in cone and plate shapes having $40 \mathrm{~mm}$ in diameter were purchased from Nakamura Glass Co Ltd., Kyoto. The nonlinear data taken with the untreated glass fixtures did not agree with the data taken by using the stainless steel fixtures in nonlinear regime due to slippage of the solution. Therefore, we first examined three types of hydrophilic surfaces, modified with chemical treatments. The first two types of surface treatments (hereafter we call A treatment and B treatment) were performed with two commercially available surface modifiers. According to the supplier, the main ingredient of the chemicals used for the A treatment is titanic oxide. The main ingredient for the $\mathrm{B}$ treatment is a water soluble polymer. The third treatment is performed with the UV ozone equipment. This treatment was used to clear up the glass surface in advance for all the 
treatments. After the analysis of these treatments, we realized that a hydrophobic surface is favorable to achieve the nonslipping measurement, and therefore we examined silane coupling treatment with trimethylchlorosilane, TMS. After the glass cone and plate were soaked in TMS, the cone and plate were rinsed with distillated water.

Contact angle measurements were preformed with DM500, Kyowa Interface Science Co., Ltd. at room temperature. The dispersion and polar portions of the surface free energy were estimated by using the Kaelble-Uy formula. ${ }^{13,14)}$ For this purpose, contact angle of two solvents, formamide and diiodomethane were measured.

\section{RESULTS AND DISCUSSION}

\subsection{Linear Viscoelasticity}

Figure 1 shows the frequency dependence of the complex shear modulus of the CTAB/NaSal aqueous solution measured with the non-treated and the A-treated glass cone-plate. After Shikata's classification, the solution is expected to be the type III solution, whose complex modulus is described with a single Maxwell model. The deviation from the Maxwell behavior at high frequencies may be attributed to polymeric (Rouse) mode of the entangled thread-like micelles.

As shown in Figure 1, the effect of surface treatments on linear viscoelastic response is not recognized. The similar agreements were obtained for all the other measurements performed with various surface treatments. Thus, the measurement of linear viscoelastic properties of the threadlike micellar solutions is insensitive to the surface treatments.

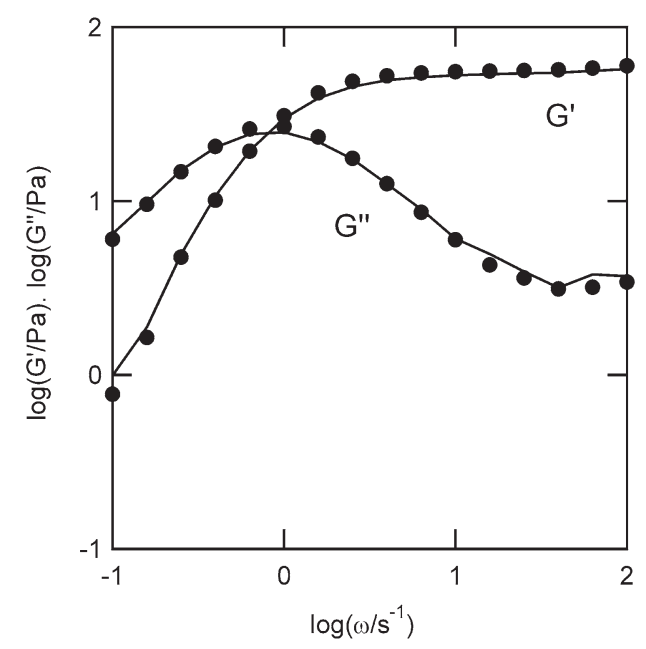

Fig. 1. Complex modulus of CTAB/NaSal solution. Filled circles represent data obtained with non-treated glassy fixture and continuous lines, the data obtained with A-treated glass fixture.
This means that the surface treatment does not affect the structure of thread-like micelles.

\subsection{Strain Hardening}

Figure 2 shows the viscosity growth function of the CTAB/ $\mathrm{NaSal}$ aqueous solution after inception of shear flow. For the case of the A-treated glass fixture, the shear stress increases with time linearly at short times, which agrees with the linear viscoelastic behavior (not shown here). Around $t / \mathrm{s}=1$, the shear stress starts to increase more rapidly and deviates from the linear viscoelastic response (strain hardening). A detailed rheological analysis is provided in the previous study. ${ }^{12)}$ The molecular origin of the strain hardening can be attributed to the finite extensibility of network strands. The strain hardening behavior observed with the A-treated glass fixture is similar to the case of the stainless steel fixture ${ }^{12)}$ except the stress maximum observed with the A-treated fixture is slightly lower than that for the latter case. After the stress maximum, the shear flow became unstable. The stress maximum is related with the slippage of the solution at the surface of the fixture. As shown in the previous study, ${ }^{12)}$ the level of stress maximum is insensitive to the rate of shear.

On the other hand, for the case of non-treated glass, the level of the stress maximum is much lower, and occurred at shorter times. Note that the stress in a range of short $t(<1 \mathrm{~s})$ agrees with that of the A treated glass. Around the stress maximum, the solution seemed to start to slip at the surface of the fixture and the flow became unstable. Figure 2 clearly indicates that the chemical treatments significantly affect the strain hardening behavior. The stress maximum values, $\sigma_{\mathrm{m}}$,

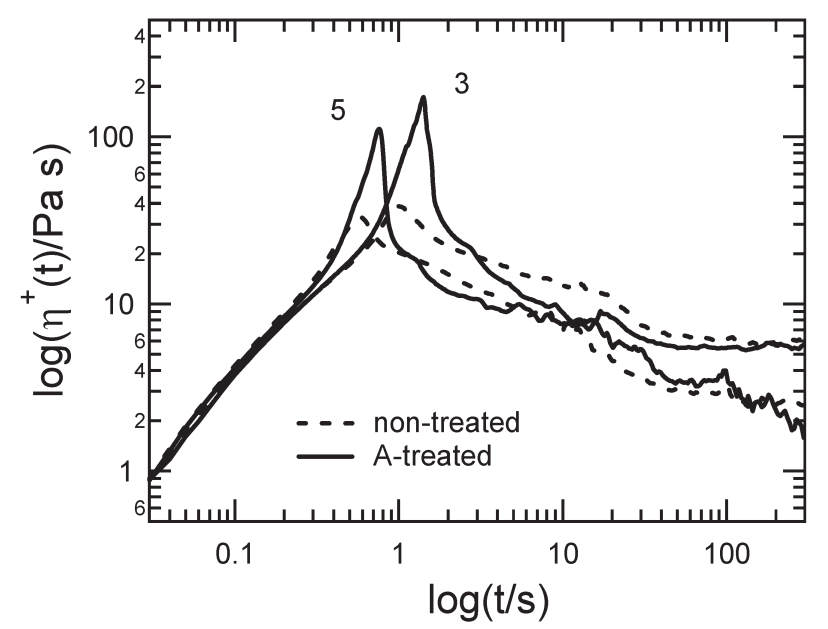

Fig. 2. Stress growth function of CTAB/NaSal solution. The A-treated glass fixture gives significant strain hardening behavior. Numbers in the figure indicate rate of shear $\left(\mathrm{s}^{-1}\right)$. 
determined with various surface treatments are summarized in Table I. We will use $\sigma_{\mathrm{m}}$ as a measure of affinity of micellar solution to the surface.

\subsection{Relationship Between Maximum Shear Stress and Contact Angle}

Contact angle measurements were often performed to estimate thermodynamic parameters of a surface. The contact angles of water to the non-, A-, and B-treated surfaces are summarized in Table I together with other properties. The value of $\sigma_{\mathrm{m}}$ cannot be simply correlated with the contact angle of water. In order to clarify the effect of surface treatment on $\sigma_{\mathrm{m}}$, we estimated the dispersion and polar components, $\gamma^{\mathrm{d}}$ and $\gamma^{p}$, of the surface free energy by using the Kaelble-Uy formula. Some details of this estimation are summarized in Appendix. The relationship between these components and $\sigma_{\mathrm{m}}$ is shown in Figure 3. With increasing $\gamma^{\mathrm{d}}$, or decreasing $\gamma^{\mathrm{p}}, \sigma_{\mathrm{m}}$ increases. Although the detailed surface structure in molecular level is not characterized, we may phenomenologically conclude that the larger dispersion component is important to obtain the good affinity of the solution to the glassy fixture.

In order to obtain the higher $\sigma_{\mathrm{m}}$ value, we designed hydrophobic surface by using silane coupling method with trimethylchlorosilane, TMS. This treatment substitutes the silanol groups of the glassy surface with trimethylsilanol groups, and therefore it is anticipated to increase $\gamma^{\mathrm{d}}$ and decrease $\gamma^{\mathrm{p}}$. Thus obtained cone and plate fixture provided the highest value of $\sigma_{\mathrm{m}}$. The surface free energy components, $\gamma^{\mathrm{d}}$ and $\gamma^{\mathrm{p}}$, were determined by the same method to clarify the relationship between $\sigma_{\mathrm{m}}$ and these components. The results were shown in Figure 3. The $\gamma^{\mathrm{d}}$ value for the TMS treated glass is not the highest. Thus, $\sigma_{\mathrm{m}}$ value may not be simply determined by the $\gamma^{\mathrm{d}}$ value. We note that the highest value of $\gamma^{\mathrm{d}}$ for the stainless steel fixture could be due to its non-negligible surface rough. It is known that the rough surfaces give larger

Table. I. Water contact angle, surface free energy values, and maximum shear stress (shear rates $=3 \mathrm{~s}^{-1}, 5 \mathrm{~s}^{-1}$ ).

\begin{tabular}{|c|c|c|c|c|c|}
\hline & \multicolumn{4}{|c|}{ Glass } & \multirow{2}{*}{ Stainless } \\
\cline { 2 - 5 } & Untreated & A treated & B treated & TMS & \\
\hline $\begin{array}{c}\text { Water contact } \\
\text { angle }\left(^{\circ}\right)\end{array}$ & 20.6 & 39.5 & 10.4 & 90 & 100.4 \\
\hline$\gamma^{\mathrm{d}} / \mathrm{mJ} \mathrm{m}^{-2}$ & 12 & 24.3 & 16.8 & 41.9 & 56.7 \\
\hline$\gamma^{\mathrm{P}} / \mathrm{mJ} \mathrm{m}^{-2}$ & 57.7 & 33.7 & 55.2 & 0.6 & 0 \\
\hline$\sigma_{\mathrm{m}} / \mathrm{Pa}\left(\right.$ for $\left.3 \mathrm{~s}^{-1}\right)$ & 115 & 519 & 444 & 1148 & 802 \\
\hline$\sigma_{\mathrm{m}} / \mathrm{Pa}\left(\right.$ for $\left.5 \mathrm{~s}^{-1}\right)$ & 165 & 558 & 504 & 1527 & 878 \\
\hline
\end{tabular}

contact angle than flat surfaces, resulting in an apparent, larger free energy. However, if we limit our discussion on the glassy fixtures having the same roughness, we can conclude that $\sigma_{\mathrm{m}}$ value seems to increase monotonically with $\gamma^{\mathrm{d}}$ value.

The present study suggests that the shear induced structure observed in glass fixture or flow channel may be different from that observed in metal fixture or flow channel. Actually, Hashimoto et al. reported that the flow curve measured with glass channel is different from that with the stainless steel. ${ }^{15}$ ) Detailed analysis of the effect of surface treatment on shearinduced structure is now under progress.

\section{CONCLUSION}

Strain hardening behavior of threadlike micellar solutions strongly depended on the surface property of the rheometer fixture. For the case of aqueous solution of CTAB and NaSal, the maximum stress in strain hardening behavior was obtained when the glass surface was treated with trimethylchlorosilane. Thus, surface treatments are very important to measure the nonlinear viscoelastic properties of threadlike micellar solutions.

\section{Acknowledgement}

This study was partially supported by a Grant-in-Aid for Scientific Research (No. 17540381) from the Ministry of Education, Culture, Sports, Science, and Technology of Japan.
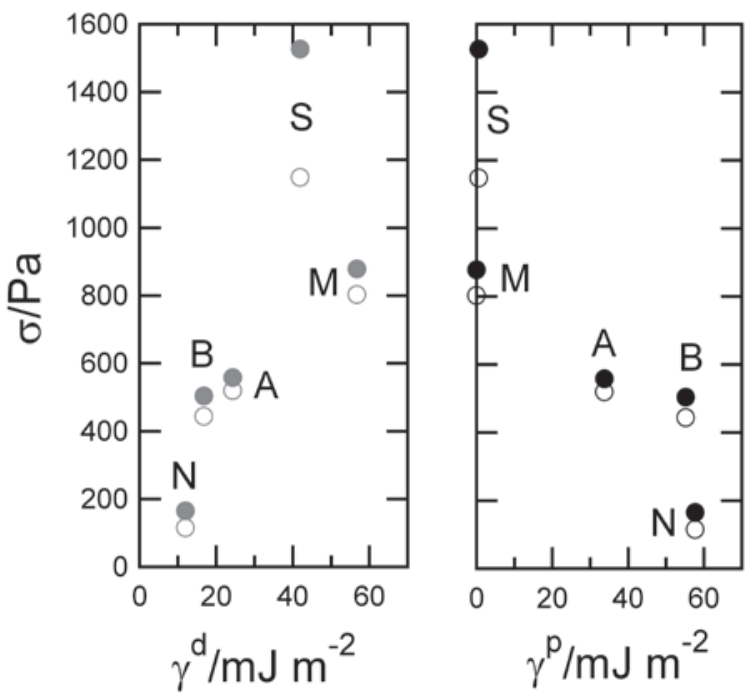

Fig. 3. Relationship between components of surface energy and maximum shear stress of stain hardening. N: not treated glass; A: A-treated glass; B: B-treated glass; S: TMS-treated glass, M: Metal (stainless steel). 


\section{APPENDIX}

When a solid comes in contact with a liquid, the reversible surface free energy change per unit area of the new interface formed, $G_{\mathrm{SL}}$ can be defined as following:

$$
G_{\mathrm{SL}}=\gamma_{\mathrm{SL}}-\gamma_{\mathrm{S}}-\gamma_{\mathrm{LV}}=-W_{\mathrm{A}}
$$

Where $\gamma_{\mathrm{S}}$ is the surface free energy of the solid in a vacuum, $\gamma_{\mathrm{LV}}$ is the surface tension of the liquid in equilibrium, $\gamma_{\mathrm{SL}}$ is the interfacial tension, and $W_{\mathrm{A}}$ is the thermodynamic work of adhesion. The equation of Young, which resolved the tensions at the interfaces (defined as $\gamma_{\mathrm{LV}}, \gamma_{\mathrm{SV}}\left(=\gamma_{\mathrm{S}}\right)$, and $\gamma_{\mathrm{SL}}$ ) of a threephase contact line, may be written by vector algebra as follows.

$$
\gamma_{\mathrm{LV}} \cos \theta=\gamma_{\mathrm{SV}}-\gamma_{\mathrm{SL}}
$$

The Young-Dupre equation is obtained by combined with eqs. A.1 and A.2.

$$
\mathrm{W}_{\mathrm{A}}=\gamma_{\mathrm{LV}}(1+\cos \theta)+\pi \mathrm{e}
$$

The $\pi_{\mathrm{e}}$ term is defined as the equilibrium osmotic pressure, and this term is commonly assumed to be zero.

The surface free energy may be divided into many components due to dispersion interactions, dipole-dipole interactions, dipole-induced-dipole interactions, hydrogen bonding, $\pi$-bonding. ${ }^{16)}$ From a practical point of view, the surface free energy of solid and liquid can be divided into two components, dispersion (depicted by ${ }^{\mathrm{d}}$ ) and polar components (depicted by ${ }^{\mathrm{p}}$ ). According to Fowkes' approach ${ }^{16)}$ extended by Owens and Wendt ${ }^{17)}$ and Kaelble and coworkers ${ }^{13,14)}$, the interface free energy of the liquid and solid phases in contact can be expressed as

$$
\gamma_{\mathrm{SL}}=\gamma_{\mathrm{S}}+\gamma_{\mathrm{LV}}-2\left(\gamma_{\mathrm{S}}^{\mathrm{d}} \gamma_{\mathrm{LV}}^{\mathrm{d}}\right)^{1 / 2}-2\left(\gamma_{\mathrm{S}}^{\mathrm{p}} \gamma_{\mathrm{LV}}^{\mathrm{p}}\right)^{1 / 2}
$$

This equation expresses the interfacial free energy as a sum of the surface free energy of phases in contact, diminished by a geometric mean of dispersion and polar interfacial interactions.

When combined with the Young-Dupre equation (A.3), and neglecting the $\pi_{\mathrm{e}}$ term, the following equation is obtained:

$$
\gamma_{L V}(1+\cos \theta)=2\left(\gamma_{S}{ }^{d} \gamma_{L V}{ }^{d}\right)^{1 / 2}+2\left(\gamma_{S}^{p} \gamma_{L V}^{p}\right)^{1 / 2}(\text { A.5) }
$$

Since the values of $\gamma_{\mathrm{LV}}{ }^{\mathrm{p}}$ and $\gamma_{\mathrm{LV}}{ }^{\mathrm{d}}$ for various liquids are listed in literature, eq. A. 5 can be solved with respect to $\gamma_{\mathrm{s}}{ }^{\mathrm{p}}$ and $\gamma_{\mathrm{s}}{ }^{\mathrm{d}}$ by measuring the contact angle for two different liquids on the same solid surface. The surface free energy, $\gamma_{S}$ is then calculated by simply adding $\gamma_{\mathrm{s}}{ }^{\mathrm{d}}$ and $\gamma_{\mathrm{s}}^{\mathrm{p}}$.

$$
\gamma_{\mathrm{S}}=\gamma_{\mathrm{S}}^{\mathrm{d}}+\gamma_{\mathrm{S}}^{\mathrm{p}}
$$

The two equations used to solve for the dispersion and polar portions of the surface free energy are obtained by taking contact angle measurements using two different liquids.

\section{REFERENCES}

1) Shikata T, Hirata H, Kotaka T, Langmuir, 3, 1081 (1987).

2) Shikata T, Hirata H, Kotaka T, Langmuir, 4, 354 (1988).

3) Shikata T, Hirata H, Kotaka T, Langmuir, 5, 398 (1989).

4) Takahashi T, Sugata H, Shirakashi M, Journal of the Society of Rheology Japan, 30, 109 (2002).

5) Shikata T, Pearson DS, In Structure and Flow in Surfactant Solutions, 1994, p 129.

6) Cappelaere E, Berret JF, Decruppe JP, Cressely R, Lindner P, Phys. Rev. E, 56, 1869 (1997).

7) Kadoma IA, vanEgmond JW, Langmuir, 13, 4551 (1997).

8) Kadoma IA, Ylitalo C, van Egmond JW, Rheo. Acta, 36, 1 (1997).

9) Hashimoto T, Turukawa T, Mori N, Nihon Reoroji Gakkaishi, 33, 1 (2005).

10) Ouchi M, Takahashi T, Shirakashi M, Nihon Reoroji Gakkaishi, 34, 229 (2006).

11) Hashimoto T, Kido K, Kaki S, Yamamoto T, Mori N, Rheol. Acta, 45, 841 (2006).

12) Inoue $T$, Inoue $Y$, Watanabe $H$, Langmuir, 21, 1201 (2005).

13) Kaelble DH, Uy KC, J. Adhes., 2, 50 (1970).

14) Kaelble DH, Cirlin EH, J. Polym. Sci., 9, 363 (1971).

15) Hashimoto $T$, Tanaka $T$, Mori N, Koshiba T, Preprint of the Rheolgoy Symposium, Japaa, 138 (2005).

16) Fowkes FM, J. Collid. Interface Sci., 493 (1968).

17) Owens DK, Wendt RC, J. Appl. Polym. Sci., 13, 1741 (1969). 